\title{
2 Soil inoculation steers restoration of terrestrial ecosystems
}

3 E. R. Jasper Wubs ${ }^{1,2,}$, Wim H. van der Putten ${ }^{1,2}$, Machiel Bosch ${ }^{3}$, T. Martijn Bezemer ${ }^{1}$

4

5

6

7

${ }^{1}$ Department of Terrestrial Ecology, Netherlands Institute of Ecology (NIOO-KNAW), P.O. Box 50, 6700 AB, Wageningen, the Netherlands. ${ }^{2}$ Laboratory of Nematology, Wageningen University and Research Centre (WUR), P.O. Box 8123, 6700 ES

Wageningen, The Netherlands. ${ }^{3}$ Vereniging Natuurmonumenten, District Zuid-West Veluwe, Planken Wambuisweg 1a, 6718 SP Ede, The Netherlands.

*Email: j.wubs@nioo.knaw.nl

Many natural ecosystems have been degraded due to human activities ${ }^{1,2}$ and need to be restored in order to protect biodiversity. However, restoration can take decades and restoration activities are often unsuccessful ${ }^{3}$ because of abiotic constraints (e.g. eutrophication, acidification) and unfavourable biotic conditions (e.g. competition or adverse soil community composition). A key question is what manageable factors prevent transition from degraded to restored ecosystems and what interventions are required for successful restoration ${ }^{2,4}$. Experiments have shown that the soil community is an important driver of plant community development ${ }^{5-8}$, suggesting that manipulation of the soil community is key to successful restoration of terrestrial ecosystems $\mathbf{s}^{3,9}$. Here we examine a large-scale, six-year old field experiment on ex-arable land and show that application of soil inocula not only promotes ecosystem restoration, but that different origins of soil inocula can steer the plant community development towards different target communities, varying from grassland to heathland vegetation. The impact of soil inoculation on plant and soil community composition was most pronounced when the topsoil layer was removed, whereas effects were less strong, but still significant, when the soil inocula were introduced into intact topsoil. Therefore, soil inoculation is a powerful tool to both restore disturbed terrestrial ecosystems and steer plant community development. 
Plants and their associated soil communities are tightly inter-linked and influence each other during successional changes in developing ecosystems ${ }^{10,11}$. Several studies with grassland plant communities have shown that inoculation with late-successional soil communities can increase the performance of late-successional target plant species, at the expense of early-successional ruderals $s^{6-8,11}$. This suggests that through inoculation with late-successional soils the typically positive feedback ${ }^{6,11}$ between late-successional plants and their soil organisms may be restored ${ }^{9}$. However, two important aspects of plant-soil community interactions have remained unexplored: first it has not yet been studied to what extent soil communities may also steer the direction of plant community development towards different target states, and second, how soil legacies affect soil inoculation success in the field is unknown. We define steering of community development as the ability to direct the species composition of communities towards different target states when starting from the same environmental conditions.

Intensive arable farming reduces diversity and simplifies food webs of soil communities ${ }^{4,12}$, and creates legacy effects in soil that can benefit weedy plant species for a long time ${ }^{4,13}$. Soil legacies are due to changes in abiotic and biotic soil conditions, for example because of increasing amounts of nutrients and soil-borne enemies of crop plants ${ }^{4}$. As part of restoration projects in Europe the soil legacy effects of arable land management are often diminished through removal of the organic topsoil down to the mineral layer underneath, which e.g. reduces soil fertility ${ }^{14,15}$. However, in many cases the ecosystems remain dominated by ruderal plant species even after the soil legacies have been removed ${ }^{7,16}$. This indicates that other constraints, such as seed availability and soil community composition, may not have been alleviated ${ }^{2,7,9}$. Restoration sites where soil legacies have been altered by topsoil removal may be particularly well-suited for testing the impact of soil inoculation on restoration with and without legacy effects of former agricultural management. 
We tested the possibility of community steering through application of soil inocula in the field by analysing a large-scale, well-replicated, soil inoculation experiment on soils that had been used for arable farming for several decades. Topsoil was removed from large (2-5 ha) spatially-separated locations. Within each of these replicate locations we inoculated two different soil inocula and established controls where no further management was implemented. We used soil inocula from two donor sites: (i) a dry heathland and (ii) a grassland that had been restored 24 years ago. After six years, we analysed plant and soil community composition, as well as abiotic soil conditions, in order to test the hypothesis that application of the different inocula would not only promote community development, but that it would also steer the development of the plant and soil community composition into the direction of the respective donor sites. We performed an additional mesocosm experiment in order to validate that soil inoculation effects would not be due to adding plant seeds only.

Soil inoculation indeed altered both plant- and soil community composition profoundly (Fig. 1, S1, S2, Table S1, S2). The composition of the plant communities in plots inoculated with heathland and grassland soils differed markedly from each other (Fig. 1). The cover of both grassland and heathland target species were promoted by both inocula, although the heathland species responded most strongly to the heathland inoculum (Fig. 2a-d). Both early- and mid-successional species remained unaffected by soil inoculation. Moreover, inoculation led to plant communities that diverged from the controls in the direction of their respective donor community (Fig. 1b, S3; Table S3). This shows that, depending on the origin of the soil inoculum, the plant community development can be steered towards either a grassland or a heathland.

Soil inoculation also drove soil community composition towards that of the donor sites (Fig 1d, f, S3; Table S3). Inoculation with heathland soil significantly increased the biomass of both bacteria and 
fungi (Table S4A). Both grassland and heathland soil inocula increased the abundance of arbuscular mycorrhizal fungi and the total number of nematodes (Table S4A). Heathland inoculum enhanced the diversity of springtails (Collembola) and mites (Acari), but not their total numbers (Table S4A). Most soil abiotic conditions were not affected by soil inoculation, except that in the inoculated soils percentage organic matter increased from 1.2 to $1.9-2.5 \%$ (Table S5A).

In the mesocosm experiment, we inoculated a common field soil (mineral subsoil from the field site) with soil inocula from the two donor sites and established controls where no inoculum was added. We placed a 2-cm layer of sterilized sand on top of the mesocosm soil in order to reduce germination from the seed bank as much as possible. We subsequently sowed a standardized mixture of 30 plant species (Table S6) in all treatments and recorded the percent cover of all plant species after 30 weeks of growth. The species mixture consists of 10 representatives each for the early-, mid- and latesuccessional stages on sandy-soils in the region. Soil inoculation with heathland and grassland soils shifted the composition of the sown plant communities in different directions (Fig. 3, S2b). Plant species representative of the target communities benefitted from soil inoculation, while early- and mid-succession species remained unaffected (Fig. 2e-h). Furthermore, the two soil inocula led to plant communities that each shifted in the direction of their respective donor community (Fig. 3).Therefore soil inoculation still steered plant community development into the direction of the vegetation composition of the donor sites, even when differences in plant propagules were excluded.

Finally, we compared the effectiveness of soil inoculation between plots with and without intact topsoil. For every treatment plot inoculated with grassland soil, we had an adjacent replicate plot where the same soil inoculum was introduced in intact topsoil with a full arable legacy. There was no such treatment for heathland soil. The plots with intact topsoil had higher soil organic matter content and nutrient availability, as well as a higher abundance of bacteria, fungi, nematodes and micro- 
arthropods (Table S4, S5). Nevertheless, soil inoculation still had a significant effect on both plant and soil community composition of the topsoil (Fig. 4, Table S8a,b). In the inoculated plots, plant species composition was closer to the donor vegetation than the respective controls, irrespective of topsoil removal (Fig. S4). However, we found that soil inoculation influenced plant and soil community composition less strongly in plots with than without intact topsoil, particularly for the soil community (Fig. 4, Table S8c).

Our results show that soil inoculation can promote ecological restoration in the field. This has been suggested before ${ }^{9}$, however, most field tests have been done with soil transplants ${ }^{14,17-21}$ and not with soil inocula ${ }^{16,22}$, which introduce considerably lower amounts of propagules. Moreover, we demonstrate that depending on the origin of the soil inoculum the restoration site could be steered to become either grassland or heathland. Glasshouse studies have indicated that the soil community is an important driver of plant community composition, and that late-successional plant species experience positive soil feedbacks, while ruderal species tend to have negative feedbacks ${ }^{6,8,11}$. However, for successful restoration plant propagules from the target community also need to be present, as many target species are dispersal limited ${ }^{23,24}$. Our mesocosm experiment shows that when differences in seed availability are alleviated, soil inoculation can still steer vegetation composition. Therefore, differences in plant community composition in the field were not solely the result of co-introducing plant propagules with the inoculum. We suggest that the co-introduction of both plant propagules and their associated soil biota restored the typically positive feedbacks among late-successional plants and their associated soil biota ${ }^{6,8,25}$. These feedbacks are thought to be major drivers of succession, suggesting that the positive effects of a single soil inoculation on the plant community may persist for prolonged periods of time. 
Our study shows that soil inoculation in the field may both promote nature restoration and steer the

community development depending on the origin of the soil inoculum. In our experiment the inocula application rates were quite different among grassland $\left(2.5 \mathrm{~L} \mathrm{~m}^{-2}\right)$ and heathland inocula $\left(1.0 \mathrm{~L} \mathrm{~m}^{-2}\right)$. However, we found the strongest effects in the treatment with the lowest amount of soil inoculum (heathland-inoculum). Therefore, we do not think that this difference was a major factor driving the results. Other studies have shown that inoculation of soils with arbuscular mycorrhizal fungi (AMF) can also promote the performance of late-successional plants over ruderal species and that local AMF strains outperform commercial strains ${ }^{25-27}$. However, several studies have argued that the transfer of whole soil communities is more effective than the addition of individual species or strains $^{25-28}$. In addition, among the soil transplantation experiments in the field ${ }^{14,16-22}$, large-sized treatments tend to be more successful at restoring native plant communities than soil transfers applied at a small spatial scale. Thinly spread soil inocula, as used in our study, can restore much larger areas than when entire soil layers are translocated ${ }^{17-20}$, which may enhance the feasibility of this procedure as soil collection will disrupt current nature areas.

The success of inoculation in the field also depended on the presence of a legacy of arable cultivation: the impact of inoculation with grassland soil was greater when the topsoil was removed than when added to intact topsoil. The treatment plots with and without arable soil legacy differed substantially in their abiotic conditions, as well as soil community composition. It is, therefore, not possible to disentangle the exact causes in this experiment. However, it has been established that both low soil nutrient conditions $\mathrm{s}^{7,29}$ and reduced competition from resident soil communities ${ }^{30} \mathrm{can}$ enhance the impact of soil inoculation. Importantly, however, the effect of soil inoculation on community composition was still clearly present even in the intact topsoil. Further research should, therefore, test whether carefully chosen soil inocula may reduce the need for expensive topsoil removal, which has considerable environmental costs ${ }^{14}$. 
153 We conclude that under field conditions soil inoculation can steer the course of community development on ex-arable land, irrespective of topsoil removal, but that effects are greatest when removing the topsoil prior to soil inoculation. Depending on the origin of the soil inoculum, the composition of the plant community in the recipient site was directed towards a heathland or a

157 grassland vegetation. Based on our results we suggest that manipulation of soil communities through 158 soil inoculation is a powerful tool for the restoration of degraded terrestrial ecosystems.

159 
The experiment was carried out on a 160 ha former arable field (Reijerscamp, the Netherlands, GPS: $52.015^{\circ} \mathrm{N}, 5.777^{\circ} \mathrm{E}$ ), which had been used to cultivate crops for almost six decades. Reijerscamp is situated on coarse Pleistocene sand (Gleyic Placic Podzol, derived from a sandur: a glacial outwash plain) with gravel and an organic top layer of $30-35 \mathrm{~cm}$, sometimes up to $50 \mathrm{~cm}$ thick. The site was grazed by cattle (25-30 cows throughout the year) upon completion of restoration measures and was further managed by periodic removal of tree seedlings (particularly Betula spp. and Prunus serotina).

Within the site, four separate experimental locations were selected. In each location the arable soil legacy was removed from 2-5 ha, by excavating the organic topsoil down to the mineral soil below (50-70 cm depth). In the excavation areas we established three treatments. We inoculated soil from two different nearby sources, a heathland and a grassland ( $H$ and $G$, respectively), in large treatment plots (on average $0.5 \mathrm{ha}$ ) in each of the four locations and control plots $(\mathrm{C})$ were established where no further treatments were executed. In addition, in order to test the impact of the organic top-layer on inoculation success, we also inoculated plots of similar size with grassland soil outside the excavations and created controls there (i.e. without topsoil removal and soil inoculation). Hence, in total there were five experimental treatments carried out in each of the four locations (i.e. $N=5 \times 4=$ 20 plots). The soil inocula were obtained from two nearby ( $<5 \mathrm{~km}$ distance) sites: one a grassland that had been under restoration for 24 years (Dennekamp, GPS: $52.029^{\circ} \mathrm{N} ; 5.801^{\circ} \mathrm{E}$ ) and an old dry heathland (Doorwerthse Heide, GPS: $51.995^{\circ} \mathrm{N} 5.778^{\circ} \mathrm{E}$ ). The grassland soil was inoculated at a rate of $\pm 2.5 \mathrm{~L} \mathrm{~m}^{-2}$ and heathland at a rate of $\pm 1.0 \mathrm{~L} \mathrm{~m}^{-2}$, with a commercial manure spreader, which resulted in a thin layer $<1 \mathrm{~cm}$.

Six years after the treatments had been implemented we conducted a field sampling campaign (July 2012, micro-arthropods: Sept 2012). To account for small-scale heterogeneity, we placed a randomly oriented transect across the centre of each of the 20 plots. Each transect consisted of five square 
subplots $\left(1 \mathrm{~m}^{2}\right)$ each separated by five meters from the next subplot (total transect length: $25 \mathrm{~m}$ ). In every subplot we analysed the composition of the plant and soil community and a number of abiotic soil parameters (see Supplementary Methods online). The same sampling was conducted in the two donor sites (July 2013) except that no data on microarthropods were collected. The two donor sites were each a priori divided into four areas of equal size using geographical stratification, and one transect was placed randomly within each area. In the heathland the selected positions of transects were slightly adjusted during sampling in the field to ensure that areas where sods had recently been cut as part of the normal management were avoided.

We analysed the data using linear mixed models for univariate response data including random effects to account for the hierarchical sampling design. We explicitly modelled heteroscedasticity in the residuals using Generalized Least Squares and post-hoc comparisons were corrected for multiple testing using the false-discovery rate. We used non-metric dimensional scaling (NMDS) to visualize differences in community composition and tested for significant differences among treatments using multiple-response permutation procedures (MRPP).

Additionally, we conducted a mesocosm experiment with mineral subsoil collected from the Reijerscamp at $>70 \mathrm{~cm}$ depth inoculated with soil from the two donor sites (9:1 w:w). Separate soil inocula were collected from each transect in the donor fields and used as replicates. We placed a layer of sterile sand on top of the soils in order to prevent germination of seeds still present in the inoculum and sowed a standardized seed mixture of 30 species (Table S6) to ensure equal seed availability. After 30 weeks of growth we recorded the cover of all species in the treatments. As the mesocosm experiment setup was qualitatively the same as the design of the field experiment we analysed the data in the same way. 
A detailed description of sample processing methods and data analysis can be found in the

Supplementary Methods online. The primary data are available in Figshare (doi: 10.6084/m9.figshare.3435404).

\section{References}

1. Vitousek, P. M., Mooney, H. A., Lubchenco, J. \& Melillo, J. M. Human domination of Earth's ecosystems. Science 277, 494-499 (1997).

2. Hobbs, R. J. \& Harris, J. A. Restoration ecology: repairing the Earth's ecosystems in the new millennium. Restoration Ecology 9, 239-246 (2001).

3. Eviner, V. T. \& Hawkes, C. V. Embracing variability in the application of plant-soil interactions to the restoration of communities and ecosystems. Restoration Ecology 16, 713-729 (2008).

4. Kulmatiski, A., Beard, K. H. \& Stark, J. M. Soil history as a primary control on plant invasion in abandoned agricultural fields. Journal of Applied Ecology 43, 868-876 (2006).

5. Van der Putten, W. H. et al. Plant-soil feedbacks: the past, the present and future challenges. Journal of Ecology 101, 265-276 (2013).

6. Kardol, P., Bezemer, T. M. \& Van der Putten, W. H. Temporal variation in plant-soil feedback controls succession. Ecology Letters 9, 1080-1088 (2006).

7. Carbajo, V., den Braber, B., van der Putten, W. H. \& De Deyn, G. B. Enhancement of late successional plants on ex-arable land by soil inoculations. PloS ONE 6, e21943 (2011).

8. De Deyn, G. B. et al. Soil invertebrate fauna enhances grassland succession and diversity. Nature 422, 711-3 (2003).

9. Harris, J. Soil microbial communities and restoration ecology: facilitators or followers? Science 325, 573-4 (2009).

10. Van der Wal, A. et al. Fungal biomass development in a chronosequence of land abandonment. Soil Biology and Biochemistry 38, 51-60 (2006). 
11. Bauer, J. T., Mack, K. M. L. \& Bever, J. D. Plant-soil feedbacks as drivers of succession: evidence from remnant and restored tallgrass prairies. Ecosphere 6, art158-art158 (2015).

12. Tsiafouli, M. A. et al. Intensive agriculture reduces soil biodiversity across Europe. Glob Change Biol 21, 973-985 (2015).

13. Kulmatiski, A. \& Beard, K. H. Long-term plant growth legacies overwhelm short-term plant growth effects on soil microbial community structure. Soil Biology and Biochemistry 43, 823-830 (2011).

14. Jaunatre, R., Buisson, E. \& Dutoit, T. Topsoil removal improves various restoration treatments of a Mediterranean steppe (La Crau, southeast France). Applied Vegetation Science 17, 236-245 (2014).

15. Marrs, R. H. in Advances in Ecological Research (eds. Fitter, A. H. \& Begon, M.) 24, 241-300 (Academic Press, 1993).

16. Kardol, P., Bezemer, T. M. \& Van Der Putten, W. H. Soil organism and plant introductions in restoration of species-rich grassland communities. Restoration Ecology 17, 258-269 (2009).

17. Box, J. et al. Experimental wet heath translocation in Dorset, England. Ecological Engineering 37, 158-171 (2011).

18. Bulot, A., Provost, E. \& Dutoit, T. A comparison of different soil transfer strategies for restoring a Mediterranean steppe after a pipeline leak (La Crau plain, South-Eastern France). Ecological Engineering 71, 690-702 (2014).

19. Vécrin, M. P. \& Muller, S. Top-soil translocation as a technique in the re-creation of species-rich meadows. Applied Vegetation Science 6, 271-278 (2003).

20. Pywell, R. F., Meek, W. R., Webb, N. R., Putwain, P. D. \& Bullock, J. M. Long-term heathland restoration on former grassland: The results of a 17-year experiment. Biological Conservation 144, 1602-1609 (2011). 
21. Antonsen, H. \& Olsson, P. A. Relative importance of burning, mowing and species translocation in the restoration of a former boreal hayfield: responses of plant diversity and the microbial community. Journal of Applied Ecology 42, 337-347 (2005).

22. Hamman, S. T. \& Hawkes, C. V. Biogeochemical and microbial legacies of non-native grasses can affect restoration success. Restoration Ecology 21, 58-66 (2013).

23. Bakker, J. P. \& Berendse, F. Constraints in the restoration of ecological diversity in grassland and heathland communities. Trends in Ecology \& Evolution 14, 63-68 (1999).

24. Bullock, J. M. Community translocation in Britain: Setting objectives and measuring consequences. Biological Conservation 84, 199-214 (1998).

25. Rowe, H. I., Brown, C. S. \& Claassen, V. P. Comparisons of mycorrhizal responsiveness with field soil and commercial inoculum for six native montane species and Bromus tectorum. Restoration Ecology 15, 44-52 (2007).

26. Emam, T. Local soil, but not commercial AMF inoculum, increases native and non-native grass growth at a mine restoration site. Restor Ecol 24, 35-44 (2016).

27. Paluch, E. C., Thomsen, M. A. \& Volk, T. J. Effects of resident soil fungi and land use history outweigh those of commercial mycorrhizal inocula: testing a restoration strategy in unsterilized soil. Restoration Ecology 21, 380-389 (2013).

28. Hoeksema, J. D. et al. A meta-analysis of context-dependency in plant response to inoculation with mycorrhizal fungi. Ecology letters 13, 394-407 (2010).

29. De Deyn, G. B., Raaijmakers, C. E. \& Van der Putten, W. H. Plant community development is affected by nutrients and soil biota. Journal of Ecology 92, 824-834 (2004).

30. Elsas, J. D. van et al. Microbial diversity determines the invasion of soil by a bacterial pathogen. PNAS 109, 1159-1164 (2012).

\section{Additional information}


Supplementary information is available online. Reprints and permissions information is available online at www.nature.com/reprints. Correspondence and requests for materials should be addressed to E.R.J.W.

\section{Acknowledgements}

We thank S. de Bruin, G. Heuvelink, and W. de Boer for their advice, and H. Hofman, H. Veerbeek, L. Veiken and A. Blankena for providing background information on the field site and the experiment. Thanks to R. Wagenaar, C. Raaijmakers, I. Chardon, H. Duyts, Q. Hakkaart, G. Bos, Y. Chau, M. Schrama, S. Schreven, J. Salamon, W. Dimmers, A. Moraru, H.-J. van der Kolk, L. Wit, and I. Nugteren for technical assistance. This work was funded by the Netherlands Organization for Scientific Research (NWO 'Biodiversiteit werkt' project nr. 841.11.008 to TMB). This is publication 6100 of the Netherlands Institute of Ecology (NIOO-KNAW).

\section{Author contributions}

M.B., T.M.B. and W.v.d.P. initiated and designed the field experiment, M.B. implemented the field experiment, E.R.J.W. and T.M.B. designed the mesocosm experiment. E.R.J.W. collected and analysed the data and wrote the first draft. All authors contributed to the final manuscript.

\section{Competing financial interests}

The authors declare to have no competing financial interests. 
307 Fig. 1. The effect of soil inoculation with two different soil inocula on plant and soil community

308 composition in the field after topsoil removal. The left column (panel a, c, e, g) shows the difference

309 in community composition (Bray-Curtis distance; range 0-1, mean \pm SE.) of the plant and soil

310 community relative to control (light bars) and the difference between plots treated with the two

311 different inocula (heathland and grassland; dark bars). Stars indicate significant differences from zero

312 (i.e. among the two communities compared per bar), while different letters above the bars indicate significant differences among means (see Table S1). The right column shows non-metric dimensional scaling (NMDS) plots (panels $b, d, f, h$ ) of community composition in the three experimental treatments and the two donor sites (dots are means, ellipses SEs). Differences among treatments were significant in all cases (Table S2). The solid arrows indicate the direction of the effect of soil inoculation on community composition. The dotted arrows indicate the distance in community composition between the inoculated plots and their respective donor community. Stress values for each NMDS analysis are given. Stress is a lack of fit statistic (zero means perfect fit) indicating to what extent the two-dimensional plot represents the multidimensional differences in community composition (Bray-Curtis distances) in the raw data. 
323 Fig. 2. Changes in abundance of plant species groups in response to soil inoculation. Shown are

324 responses of target species for grassland $(a, e)$ and heathland $(b, f)$ respectively, as well as early- (c, $g$ )

325 and mid-succession ( $d, h)$ species. The top panels represent data from the field experiment (a-d),

326 while the bottom panels are from the mesocosm experiment (e-h). Different letters indicate

327 significant differences, for the overall analysis see Table S9. See Fig S2 for species membership to the 328 different successional groups. 
Fig. 3. Differences in plant community composition from the control due to inoculation $(10 \% \mathrm{w}: \mathrm{w})$ with grassland and heathland soil inocula respectively in a controlled mesocosm experiment. To

331 alleviate differences in seed availability, inoculated and non-inoculated containers were sown with a 332 standardized seed mixture of 30 plant species (Table S6) and a layer of sterilized sand was placed 333 over the mesocosm soil to prevent germination from the seed bank. a, The difference in community composition (Bray-Curtis distance; range $0-1$, mean \pm SE) of the vegetation relative to control (light bars) and the difference between mesocosms treated with the two different inocula (heathland and grassland; dark bar) are shown. Stars indicate significant difference in community composition (i.e. different from zero; Table S7). b, Visualization of the differences in plant community composition (dots are means, ellipses SEs) using non-metric multidimensional scaling (NMDS; MRPP Pseudo-F = 4.1, $p=0.001)$. For each of the mesocosms their similarity to the two donor sites was calculated, and the direction of increasing similarity is plotted using dashed arrows. The solid arrows indicate the direction of the effect of soil inoculation on community composition. 
342 Fig. 4. Comparison of the effect of soil inoculation on plant (a) and soil community (b-d)

343 composition in plots with (light bars) and without (dark bars) topsoil removal. The extent to which

344 the communities were different from the control (mean \pm SE, Bray-Curtis dissimilarity; range 0-1) after

345 inoculation with soil from a heathland and a grassland is shown. Data in the light bars show the effect

346 of inoculation with heathland and grassland soil after removal of the topsoil, data in the dark bars is

347 the effect of inoculation with grassland soil in plots where the topsoil had not been removed. Stars

348 indicate significant differences from zero (which equals no effect), different letters indicate

349 significant differences among means within panels (see Table S8 for analysis). 

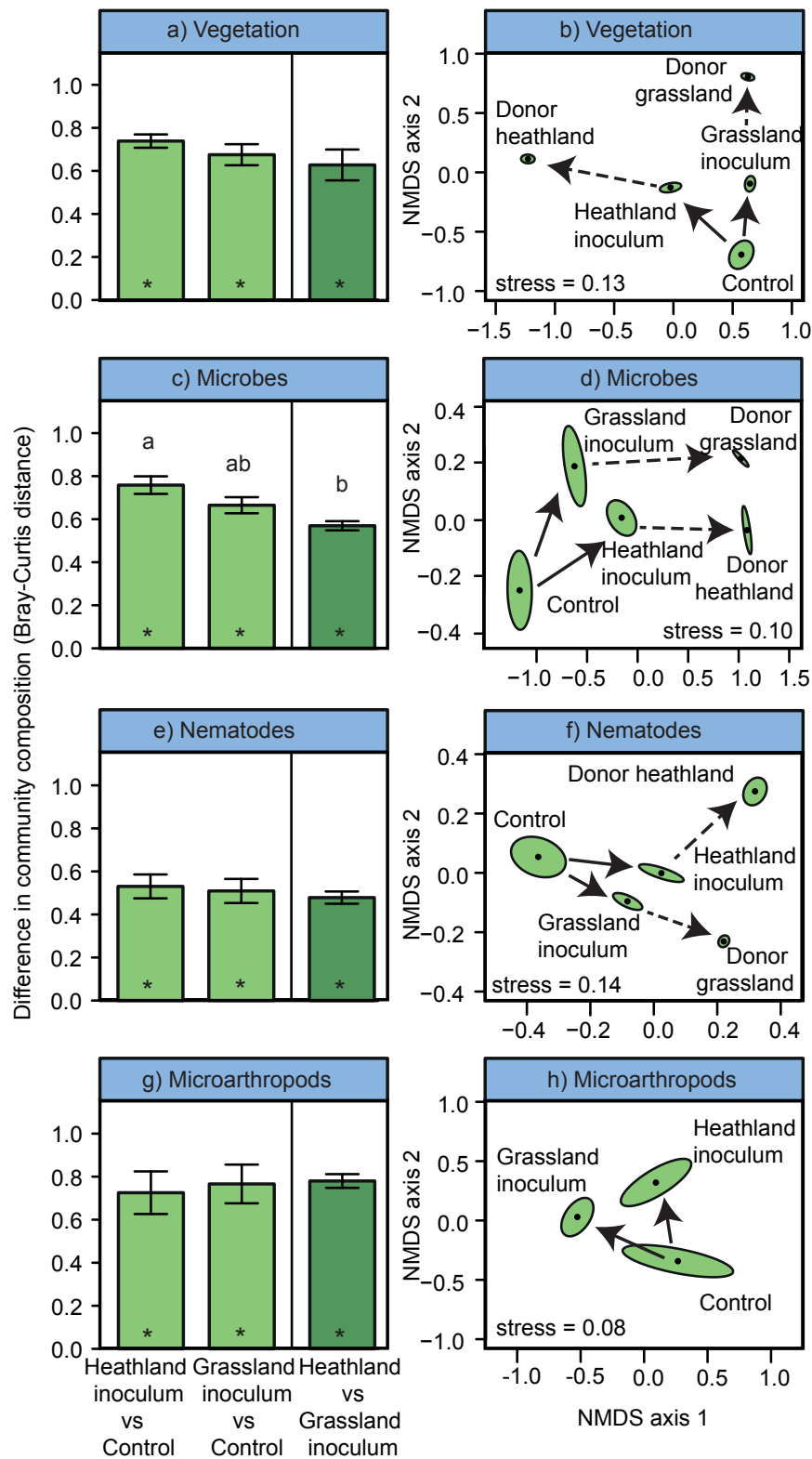


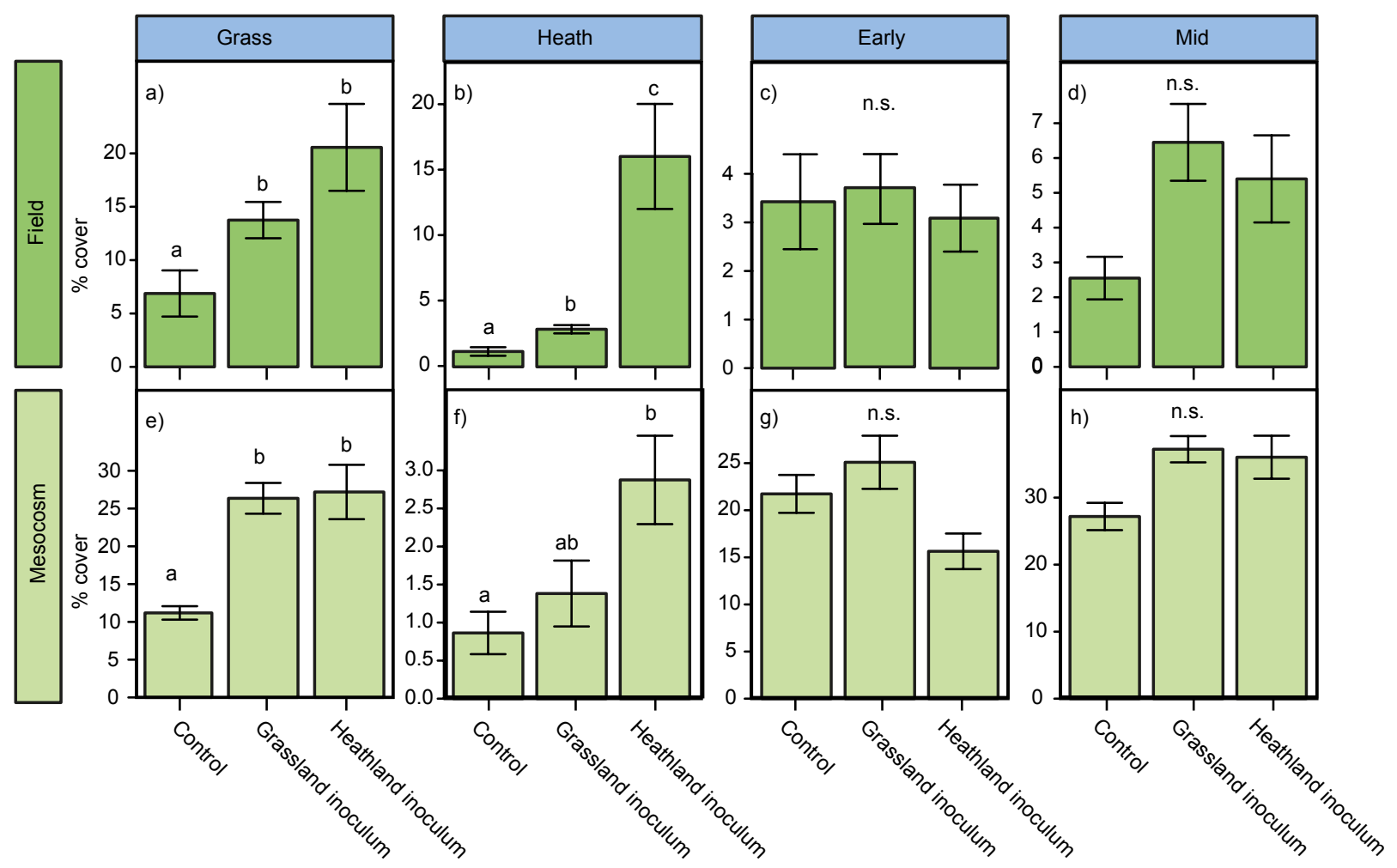




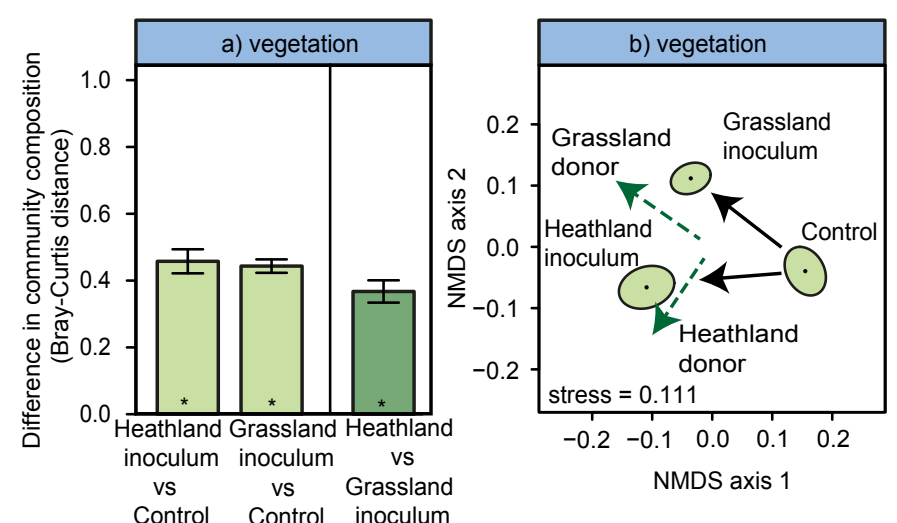




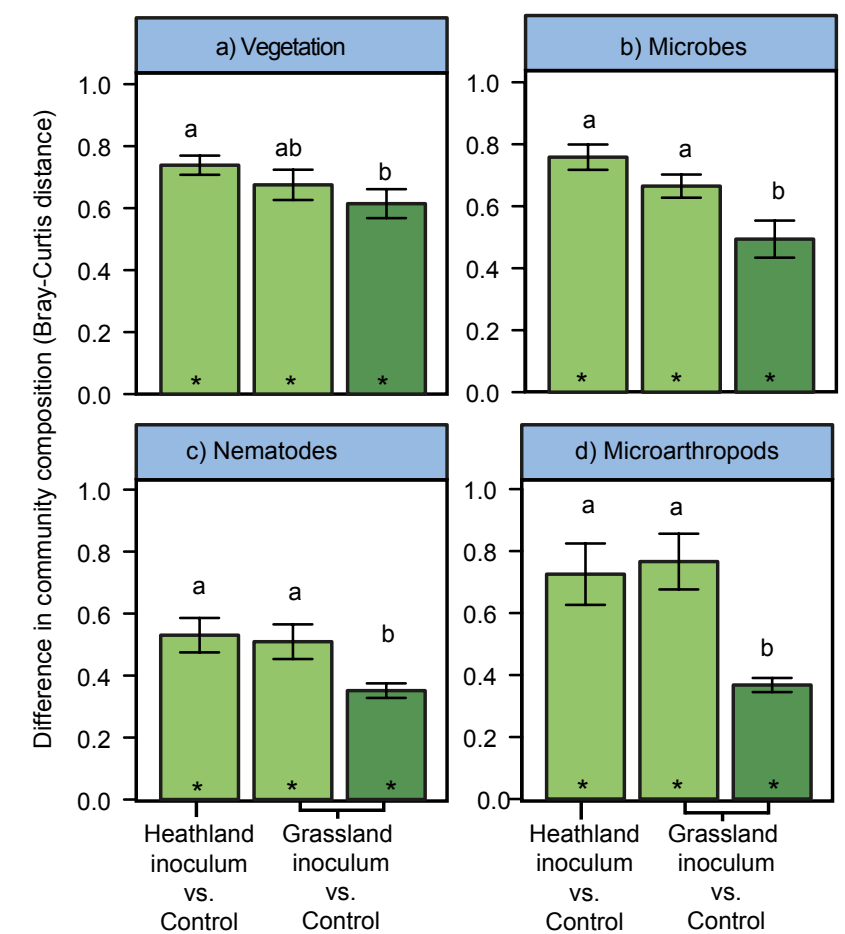

